\title{
Report
}

\section{Monotherapy efficacy of BBB-permeable small molecule activators of PP2A in}

\section{glioblastoma}

Joni Merisaari $^{1,2}$, Oxana V. Denisova ${ }^{1}$, Milena Doroszko ${ }^{3}$, Vadim Le Joncour ${ }^{4}$, Patrik Johansson ${ }^{3}$, William P.J. Leenders ${ }^{5}$, David B. Kastrinsky, ${ }^{6,7}$, Nilesh Zaware ${ }^{6}$, Goutham Narla ${ }^{8}$, Pirjo Laakkonen $^{4,9}$, Sven Nelander ${ }^{3}$, Michael Ohlmeyer ${ }^{6,10}$, Jukka Westermarck ${ }^{1,2, \#}$

${ }^{1}$ Turku Bioscience Centre, University of Turku and Åbo Akademi University, Turku, Finland ${ }^{2}$ Institute of Biomedicine, University of Turku, Turku, Finland

${ }^{3}$ Department of Immunology Genetics and Pathology, Uppsala University, Uppsala, Sweden

${ }^{4}$ Translational Cancer Medicine Research Program, Faculty of Medicine, University of Helsinki, Helsinki, Finland;

${ }^{5}$ Radboud Institute for Molecular Life Sciences, Department of Biochemistry, Nijmegen, The Netherlands

${ }^{6}$ Icahn School of Medicine at Mount Sinai, New York, New York, USA

${ }^{7}$ Donald and Barbara Zucker School of Medicine at Hofstra/Northwell, Hempstead, NY, USA

${ }^{8}$ Department of Internal Medicine, Division of Genetic Medicine, University of Michigan, Ann Arbor, MI, USA

${ }^{9}$ Helsinki Institute of Life Science - HiLIFE, Laboratory Animal Centre, University of Helsinki, Helsinki, Finland

${ }^{10}$ Atux Iskay LLC, Plainsboro, NJ, USA

The total character count: 22131

\#Corresponding author: Jukka Westermarck, tel. $+358-29-450$ 2880, jukka.westermarck@bioscience.fi 


\section{Abstract}

Glioblastoma (GB) is a fatal disease in which most targeted therapies have clinically failed. However, pharmacological reactivation of tumor suppressors has not been thoroughly studied as yet as a GB therapeutic strategy. Tumor suppressor Protein Phosphatase 2A (PP2A), is inhibited by non-genetic mechanisms in GB, and thus it would be potentially amendable for therapeutic reactivation. Here we demonstrate, that small molecule activators of PP2A (SMAPs), NZ-8-061 and DBK-1154, effectively cross the in vitro model of blood-brain barrier (BBB), and in vivo partition to mouse brain tissue after oral dosing. In vitro, SMAPs exhibit robust cell killing activity against five established GB cell lines, and nine patient-derived primary glioma cell lines. Collectively these cell lines have heterogenous genetic background, kinase inhibitor resistance profile, and stemness properties; and they represent different clinical GB subtypes. Oral dosing of either of the SMAPs significantly reduced growth of infiltrative intracranial GB tumors. DBK1154, with both higher degree of brain/blood distribution, and more potent in vitro activity against all tested GB cell lines, also significantly increased survival of mice bearing orthotopic GB xenografts. In summary, this report presents a proof-of-principle data for BBB-permeable tumor suppressor reactivation therapy for glioblastoma cells of heterogenous molecular background. 


\section{Introduction}

One of the hallmarks of GB is dysregulated phosphorylation-dependent pathways, which are drivers of its malignant progression (Dunn et al., 2012). Nevertheless, all tested kinase inhibitors have failed to prolong the overall survival of GB patients in clinical trials (Mooney et al., 2019; Tomiyama and Ichimura, 2019). One of the reasons is that the blood-brain barrier (BBB) prevents the otherwise potentially effective kinase inhibitors from reaching the brain at high enough concentrations (Harder et al., 2018). Another potential reason is non-mutational plasticity induced by kinase inhibitors in GB cells (van den Heuvel et al., 2017). Notably, phosphorylation of GB driver pathways is not only regulated by kinases, but also by phosphatases (Narla et al., 2018; Tomiyama et al., 2019). Amongst them, Protein phosphatase 2A (PP2A) is a serine/threonine phosphatase that regulates multiple oncogenic kinase signaling pathways, as well as apoptotic mechanisms (Perrotti and Neviani, 2013; Kauko and Westermarck, 2018). Interestingly, because PP2A is not genetically inactivated in GB (Kaur et al., 2016), it could be a suitable target for tumor suppressor reactivation therapies in this medically challenging indication.

A series of small molecule activators of $\underline{\text { PP}} 2 \mathrm{~A}$ (SMAPs), was recently derived from tricyclic neurological drugs such as Chlorpromazine and Clomipramine (Kastrinsky et al., 2015). Based on photoaffinity labeling studies, radiolytic footprinting, and mutagenesis of the binding site, the target of SMAPs has been identified as the interface of the A and C subunits in the PP2A complex (Sangodkar et al., 2017). The SMAPs have shown efficacy as orally available monotherapy in animal models in solid non-CNS cancer types (Sangodkar et al., 2017; Kauko et al., 2018), but 
neither their BBB penetration properties, nor potential as CNS cancer drug has not been studied as yet.

\section{Results and discussion}

\section{Development of blood-brain barrier permeable small molecule reactivators of PP2A}

We sought to improve the potency and oral bioavailability of RTC-5 and RTC-30 (Kastrinsky et al., 2015), earlier members of the SMAP series derived from tricyclics, by constraint of the linear spacer moiety between the tricyclic and sulfonamide, resulting in structures exemplified by DBK1154 and NZ-8-061 (Fig. 1A, and Supplementary figure 1). However, it was unclear whether the polarity introduced to tricyclics by the sulfonamide and hydroxyl moieties (Fig. 1A) would compromise BBB permeability, and eventually CNS availability, of the SMAPs as compared to tricyclics. We therefore started by investigating the in vitro BBB passage of NZ-8-061 (a.k.a DT061) that has been widely used in cancers outside the CNS (Sangodkar et al., 2017; Kauko et al., 2018; McClinch et al., 2018). Quantified by HPLC-MS/MS, NZ-8-061 was found to cross the artificial BBB (Le Joncour et al., 2019), consisting of murine brain microcapillary endothelial cells and astrocytes (Fig. 1B,C). Further, 24-hour pre-treatment with NZ-8-061 did not modify diffusion of a low molecular weight fluorescent probe, the sodium fluorescein $(\mathrm{Na}-\mathrm{Fl})$, indicating that passage of NZ-8-061 was not a bystander effect due to its effects on BBB model permeability (Fig. 1D). 
To study brain penetration of NZ-8-061 in vivo, we performed a pharmacokinetic study by either administering $1 \mathrm{mg} / \mathrm{kg}$ i.v. or a bolus oral dose of $100 \mathrm{mg} / \mathrm{kg}$. The in vivo pharmacokinetic parameters are shown in Figure 1E. NZ-8-061 shows $100 \%$ oral bioavailabilty based on dose adjusted fraction absorbed $(\% \mathrm{~F})$ and moderate clearance as judged from half-life in plasma with $\mathrm{T}_{1 / 2}$ of $3 \mathrm{hr}$ after I.V. dose. Peak plasma concentration after oral dose is around 14 micromolar and combined with moderate clearance and high AUC, shows significant, and sustained systemic exposure. Importantly, based on HPLC-MS/MS analysis from the whole brain homogenate, NZ8-061 partitions into brain with a brain/plasma ratio of 1:1 at 6 hours post drug administration (Fig. $1 \mathrm{E})$.

Together these results identify NZ-8-061 as an orally bioavailable, and BBB-permeable drug candidate for GB treatment. 
A
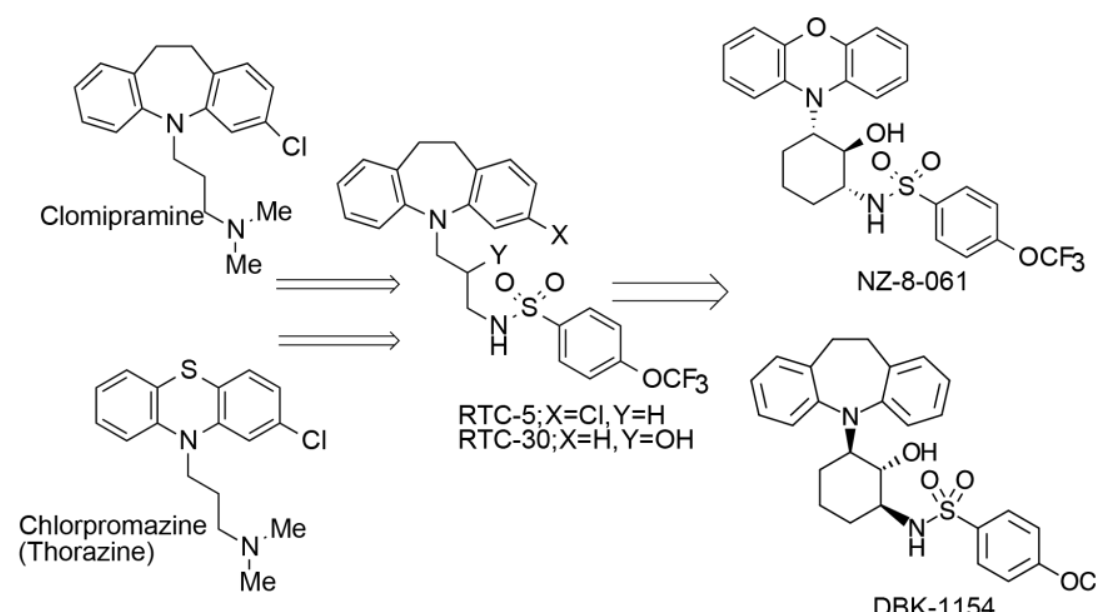

B

C
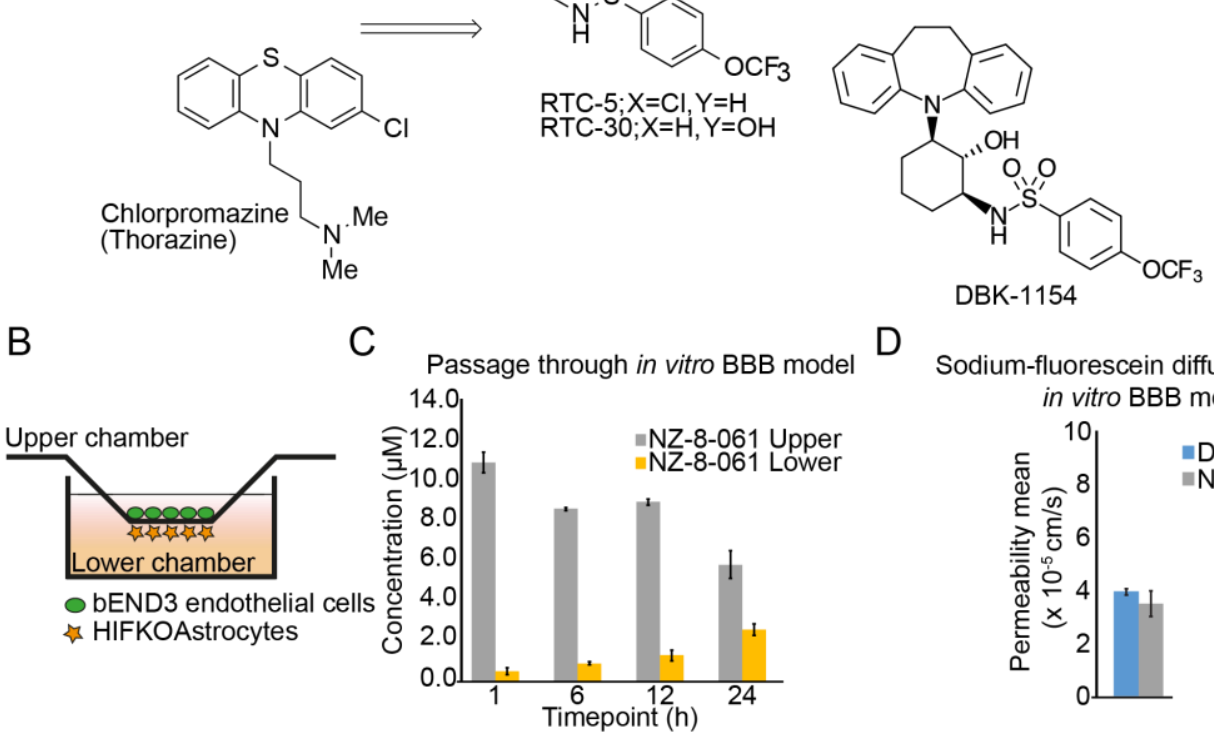

C

D

Sodium-fluorescein diffusion through

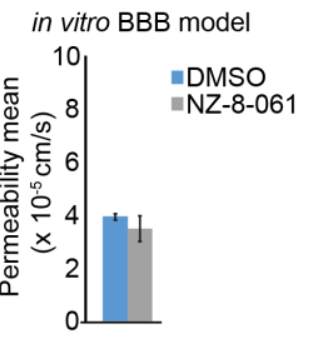

E

\begin{tabular}{|c|c|c|c|c|c|c|c|c|c|}
\hline Compound ID & Route & Dose & $\begin{array}{c}\mathrm{T} / 2 \\
\mathrm{hr}\end{array}$ & $\begin{array}{c}\mathrm{Tmax} \\
\mathrm{hr}\end{array}$ & $\begin{array}{c}\mathrm{Cmax} \\
\mathrm{ng} / \mathrm{mL}\end{array}$ & $\begin{array}{c}\mathrm{AUC} \\
\mathrm{hr} . \mathrm{ng} / \mathrm{mL}\end{array}$ & $\begin{array}{c}\mathrm{CL} \\
\mathrm{mL} / \mathrm{hr} / \mathrm{kg}\end{array}$ & $\% \mathrm{~F}$ & $\begin{array}{c}\text { Blood- } \\
\text { brain } \\
\text { ratio }\end{array}$ \\
\hline $\mathrm{NZ-8-061}$ & i.v. & $1 \mathrm{mg} / \mathrm{kg}$ & 3.3 & - & - & 858 & 970 & & \\
\hline NZ-8-061 & p.o. & $100 \mathrm{mg} / \mathrm{kg}$ & 4.4 & 4.0 & 7127 & 93367 & - & 100 & $1: 1$ \\
\hline
\end{tabular}

For $\mathrm{Mr}=500 \mathrm{~g} / \mathrm{mol}, 1000 \mathrm{ng} / \mathrm{mL}=2.0$ micromolar

Figure 1

\section{Figure 1: Blood-brain-barrier penetrance and mouse in vivo pharmacokinetics of NZ-8-061}

A) An overview of development of small molecule activators of PP2A, NZ-8-061 and DBK-1154. The synthesis is described in detail in supplementary figure 1. B) Schematic presentation of in vitro BBB model which consists of murine endothelial cells and astrocytes as described in detail in (29). C) NZ-8-061 passage through the in vitro BBB model after addition of $15 \mu \mathrm{M}$ dosage on the upper chamber at indicated timepoints. Data shown are means from two replicates \pm SD. D) Sodium-fluorescein diffusion through the in vitro BBB after 24 hour of pretreatment with $15 \mu \mathrm{M}$ NZ-8-061 on the upper chamber. Fluorescence signal of sodium-fluorescein was measured from lower chamber after 15 minutes. Data shown are means from two replicates \pm SD. E) Mouse in vivo pharmacokinetic parameters $\left(\mathrm{T}^{1} 12 \mathrm{hr}\right.$, Tmax hr, Cmax ng/mL, AUC hr.ng/mL, CL mL/hr/kg, \%F and blood-brain ratio) after $1 \mathrm{mg} / \mathrm{kg}$ or $100 \mathrm{mg} / \mathrm{kg}$ dosage via p.o. or i.v. of NZ-8-061. 


\section{NZ-8-061 potently inhibits the viability of GB cells with heterogenous genetic background}

Frequency of genetic mutations or deletions in any of the genes coding for core PP2A complex components in GB clinical isolates is negligible (Kaur et al., 2016). However, we found that all studied GB cell lines expressed higher levels of PP2A inhibitor proteins (PAIPs); PME-1, CIP2A, SET, and ARPP-19 (Kauko and Westermarck, 2018), as compared to non-tumor fibroblasts (Fig. 2A). These results provide a non-genetic candidate mechanism for PP2A deregulation in GB cells.

Genomic heterogeneity and stemness characteristics are known to affect GB cell therapy responses. Genomic characteristics of the U87MG, A172, U118 and T98G cell lines were examined using the Cancer Cell Line Encyclopedia (CCLE) (Barretina et al., 2012) database, whereas for the E98 cells published data was used (Claes et al., 2008; Navis et al., 2015). Collectively, the cell lines displayed a variety of known genomic alterations in GB (Supplementary Fig. 2A). However, the only common genomic change across all cell lines was copy number loss of CDKN2A (Supplementary Fig. 2A). Related to stemness properties, most of the established cell lines, except for E98, cultured with bovine serum supplements did not express any detectable levels of glioma stem cell markers SOX2 or Nestin (Fig. 2B).

We previously demonstrated notable kinase inhibitor resistance of T98G cells across different kinase families (Kaur et al., 2016). To further examine kinase inhibitor responses of the used cell lines, we extracted the IC50 values for selected inhibitors from Genomics of Drug Sensitivity in Cancer database (https://www.cancerrxgene.org/). No information for E98 cell line was available. 
Consistently with their heterogenous mutation profiles (Supplementary Fig. 2A), each of the cell line have very variable sensitivity against different kinase inhibitors (Supplementary Fig. 2B). We experimentally validated Gefitinib resistance of three of the cell lines (Supplementary Fig. 2C).

These genetically diverse cell lines, displaying differential drug sensitivities, were then screened for their response to PP2A reactivation by NZ-8-061 in a cell viability assay. NZ-8-061 did induce a dose-dependent reduction in cell viability in all of the tested cell lines (Fig. 2C). Opposite to their kinase inhibitor responses, neither drastic differences, nor correlations of response with their genetic background, were found among the tested cell lines based on their IC50s. As a pharmacologic control, U87MG, U118, or A172 cells

were also treated with increasing doses of TRC-766, a structurally similar but biologically inactive derivate of SMAPs (Supplementary Fig. 3A)(Sangodkar et al., 2017; McClinch et al., 2018). While TRC-766 still binds PP2A, it is unable to reactivate PP2A even at a concentration of $20 \mu \mathrm{M}$ in vitro (Sangodkar et al., 2017). Notably, TRC-766 did not affect the viability of GB cells at concentrations up to $40 \mu \mathrm{M}$ (Supplementary Fig. 3B). This strongly supports a dependence on PP2A reactivation for NZ-8-061-elicited killing of GB cells. Using a colony growth assay, NZ-8061 treatment resulted in dose-dependent growth inhibition in all of the cell lines tested and in approximately the same concentration range as seen in the cell viability assay (Fig. 2D,E). Of note, micromolar dosing of SMAP is consistent with micromolar concentration of its molecular target PP2A in cancer cells, and required as serum binding of these compounds decreases their apparent potency in standard cell culture medium. 
To extend these observations, we tested the therapeutic potential of NZ-8-061 in a series of thoroughly validated patient-derived primary glioma cells (Xie et al., 2015). Notably, chosen cells represented all three molecular subtypes of GB according to Verhaak and colleagues (Wang et al., 2017) (Supplementary Fig. 4A,B). Each of the patient-derived glioma cell line, cultured in serumfree neural stem cell media for the maintenance of stemness properties, expressed Nestin and Sox2, as well as PAIPs (Supplementary Fig. 4C and Fig. 2F). Further, they have a diverse genetic background (Supplementary Fig. 5A,B), and kinase inhibitor responses (Supplementary Fig. 6). All nine tested primary glioma cell lines displayed near to complete suppression of cell viability when treated with $5 \mu \mathrm{M}$ NZ-8-061 (Fig. 2G). The lower working concentration of NZ-8-061 as compared to standard GB cell line cultures could be explained by the lack serum in the neural stem cell media, thus increasing the apparent potency of NZ-8-061.

Together these data demonstrate that SMAPs, as exemplified by NZ-8-061, have wide-spectrum therapeutic effect in vitro across human GB cells; regardless of their genetic background, disease subtype, or stemness properties. 


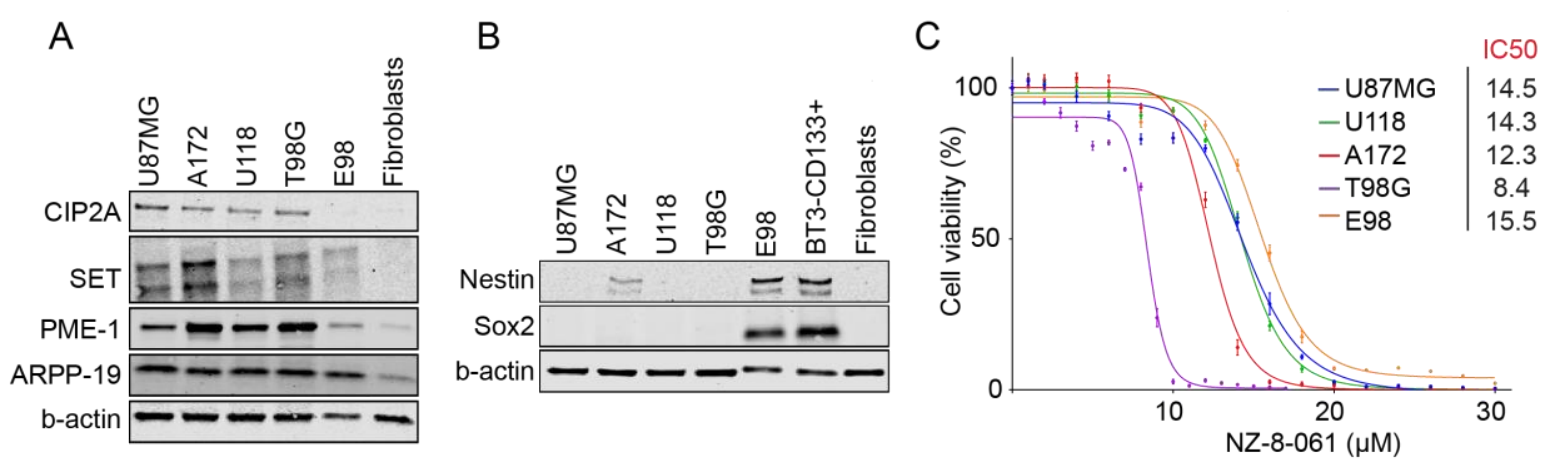

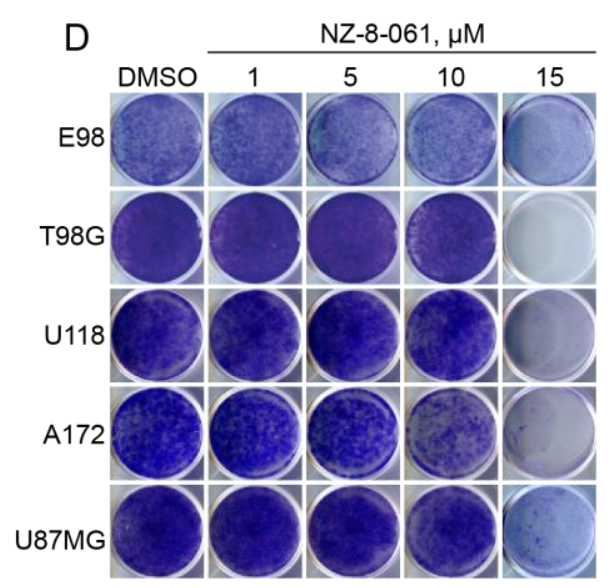

$\mathrm{F}$

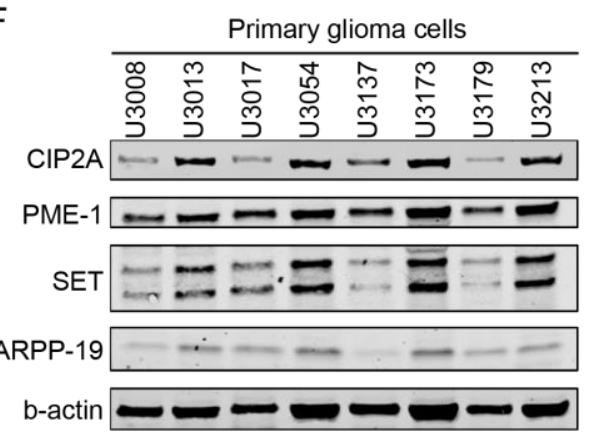

E

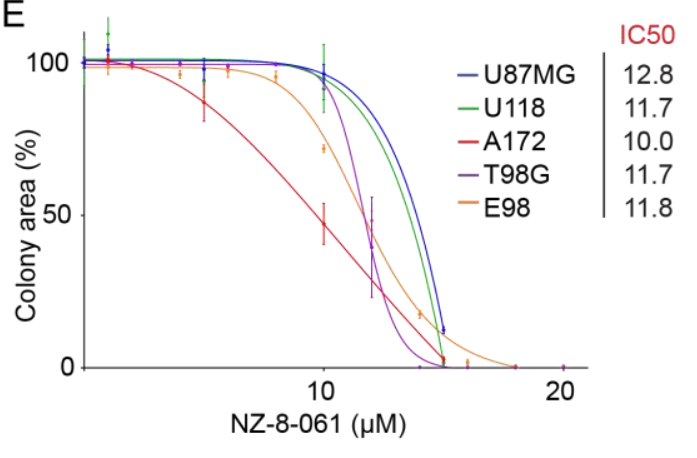

G

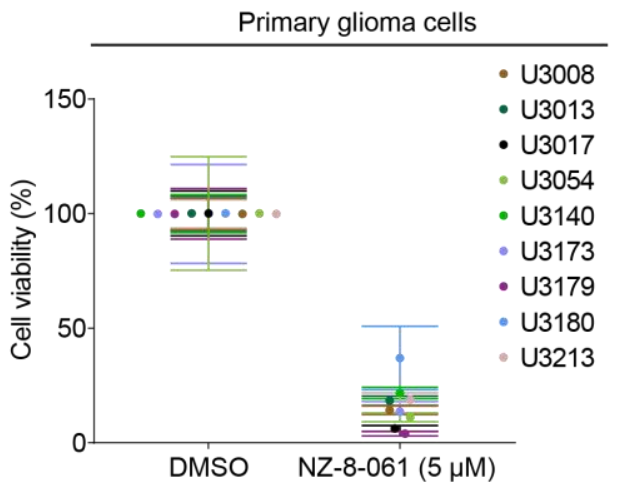

Figure 2

Figure 2: NZ-8-061 potently inhibits viability of molecularly heterogenous GB cells in vitro.

A) Western blot of endogenous PP2A inhibitor proteins (CIP2A, SET, PME-1 and ARPP-19) and B) GB stem cell markers (Sox2 and Nestin) in indicated GB cell lines. BT3-CD133+ (BT3 patient derived cells sorted for CD133) cells were used as a control for GB stem cell markers, and human fibroblasts were used as a negative control cell line in both. C) The dose-dependent effect of NZ-8-061 on the viability of indicated GB cell lines after 72 hours. Data shown are means from 6 replicates \pm SD. IC50 values were calculated with GraphPad Prism 8. D, E) Colony growth reduction after NZ-8-061 treatment in GB cell lines with indicated concentrations. IC50 values for each cell line are shown. Data shown are means from 4 replicates \pm SD. IC50 values were calculated with GraphPad Prism 8. F) Expression levels endogenous PP2A inhibitor proteins (CIP2A, SET, PME-1 and ARPP-19) in patient-derived primary glioma cells. G) Cell viability inhibition after NZ-8-061 $(5 \mu \mathrm{M})$ treatment in patient-derived primary glioma cells. Data shown are means from 6 replicates $\pm \mathrm{SD}$. 


\section{Preclinical activity of NZ-8-061 in an infiltrative intracranial GB model}

To analyze in vivo therapeutic potential of oral dosing of NZ-8-061, we used an intracranial GB tumor model with luciferase-expressing/bioluminescent E98 cells (Claes et al., 2008). Among the cell lines, E98 cells were selected as a model due to their stem-like properties (Fig. 2B), and infiltrative growth pattern in mouse brain recapitulating the human malignant glioma histology (Claes et al., 2008) (Fig. 3A). Prior to the treatment, mice were randomized into two groups based on the tumor bioluminescence signal and using a protocol for optimized design and analysis of preclinical intervention studies in vivo (Laajala et al., 2016). NZ-8-061 was thereafter orally dosed twice a day at $30 \mathrm{mg} / \mathrm{kg}$. Based on pharmacokinetics and tissue distribution (Fig. 1E), the brain exposure of NZ-8-061 was estimated to transiently reach $10 \mu \mathrm{M}$, which is roughly comparable to the IC50 for colony formation assay with NZ-8-061 in vitro (Fig. 2E).

Intracranial tumor growth was followed by bioluminescence measurements every third day. Notably, NZ-8-061 induced tumor growth stasis at day 12 and displayed a significant reduction in tumor size at later timepoints (Fig. 3B). The therapeutic response was validated by significant inhibition in tumor cell Ki67 expression in the end-point tumors when comparing treated and nontreated groups (Fig. 3C, D). 
A

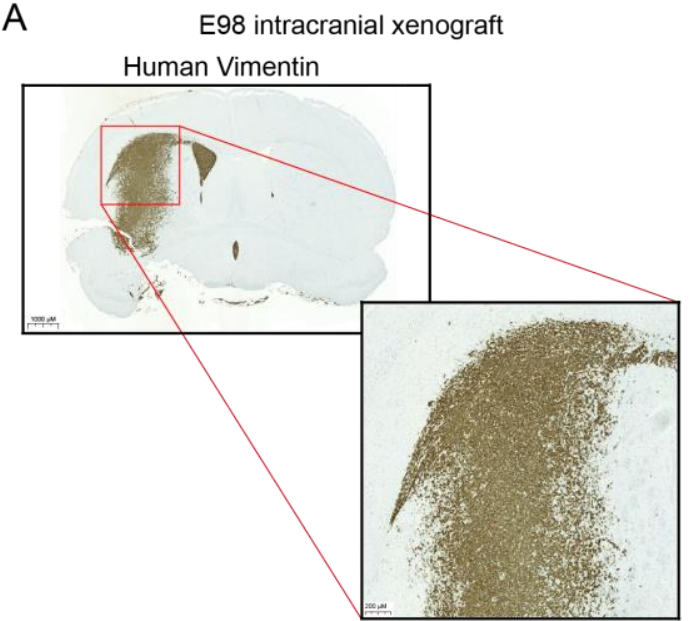

C

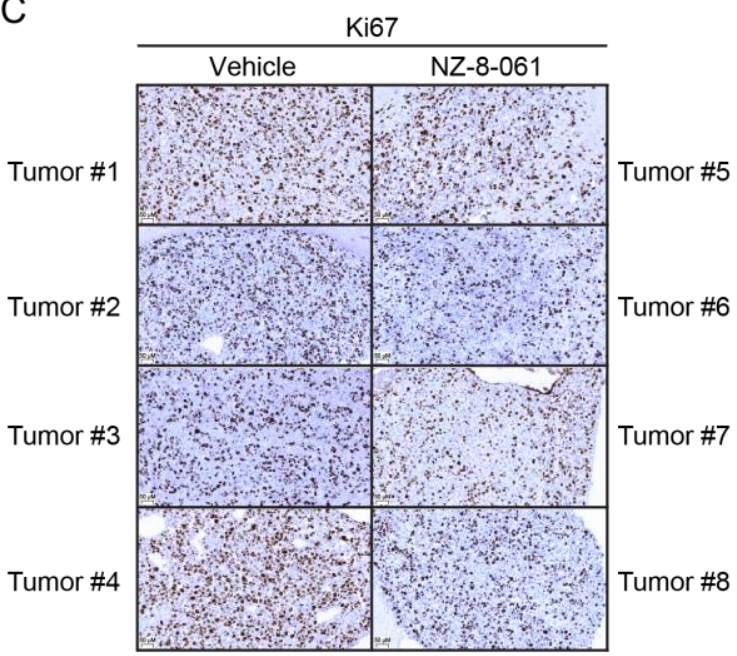

B E98 intracranial xenograft

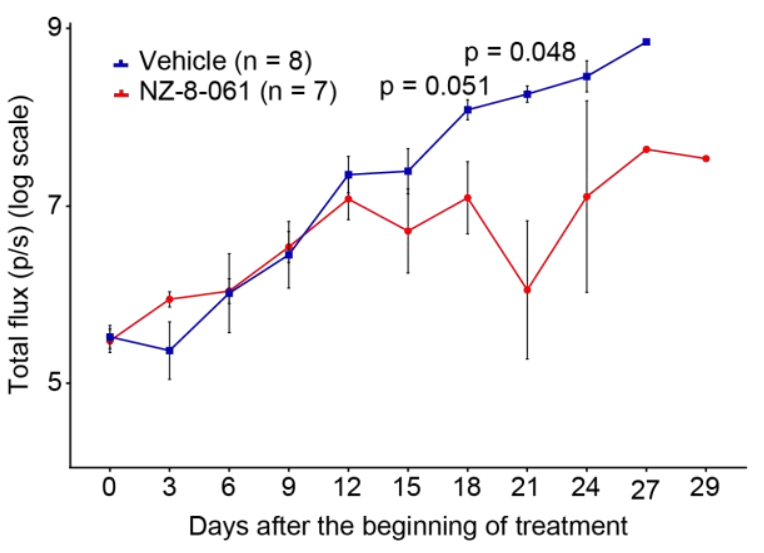

D

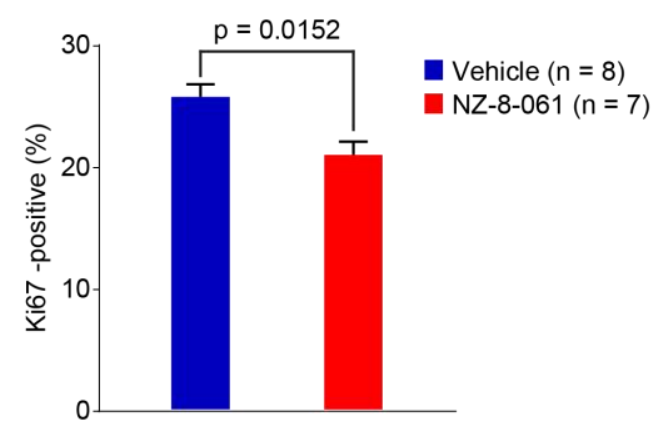

Figure 3

Figure 3: Therapeutic potential of oral dosing of NZ-8-061 as monotherapy in an infiltrative intracranial GB mouse model.

A) Example picture of infiltrative growth of intracranial human E98-FM-Cherry cell line xenograft. The mouse brain tissue was stained with human specific vimentin antibody. B) Bioluminescence follow up of the intracranial human E98-FM-Cherry cell line xenograft growth during vehicle or $30 \mathrm{mg} / \mathrm{kg}$ NZ-8-061 treatment. When tumors were visible with bioluminescence, mice were randomized for either vehicle or NZ-8-061 groups. Data shown are means from 8 replicates \pm SEM, p-values by Student's t-test. C) Representative images of Ki-67 staining from 4 vehicle and 4 NZ-8-061-treated end-point tumors from B). D) Quantification of Ki-67 positivity from C). Data shown are means of $\%$ of Ki-67 positive tumor cells from 7-8 tumors/group \pm SD, *, p-value by Mann-Whitney test. 


\section{DBK-1154, with higher degree of brain/blood distribution, and more efficient in vitro activity, increases survival of mice bearing orthotopic GB tumors}

Despite its significant in vivo efficacy in reducing intracranial tumor growth with roughly IC50 dosing (Fig. 3B-D), NZ-8-061 monotherapy failed to improve the mouse survival (Supplementary figure 7A). We therefore next tested the BBB permeability and GB cell killing properties of DBK1154. DBK-1154, with a dibenzoazepine tricyclic, has a hydrocarbon bridge versus an oxygen bridge in NZ-8-061 (Fig. 1A), making DBK-1154 somewhat more lipophilic. The calculated LogP (log octanol-water partition coefficient), a measure of lipophilicity, is higher for DBK-1154 (cLogP 7.0) than NZ-8-061 (cLogP 6.6). Additionally, tPSA (total polar surface area) is higher for NZ-8-061 versus DBK-1154 (88 vs $79 \mathrm{~A}^{2}$ ). Lower tPSA and higher cLogP generally correlate with higher CNS distribution (Kelder et al., 1999).

In vivo pharmacokinetic parameters for DBK-1154 in mouse are shown in figure $4 \mathrm{~A}$ and in supplementary figure 7B. DBK-1154 is orally bioavailable, however it showed significantly lower systemic plasma exposure compared to NZ-8-061. Importantly, in addition to in vitro BBB permeability (Supplementary Fig. 8A), the in vivo evaluation of distribution of DBK-1154 into the CNS showed a 2.3-fold higher concentration in brain tissue versus plasma (Fig. 4A).

Based on the IC50-value, higher potential of DBK-1154 was seen in a long-term colony growth assay in which the IC50 for DBK-1154 was almost two-fold lower than that of NZ-8-061 in both cell lines (Fig. 4D, insert). In addition, DBK-1154 was qualitatively different from NZ-8-061 in 
terms of its apoptosis inducing potential. With single $13 \mu \mathrm{M}$ dosing, only DBK-1154 induced Caspase-3/7 cleavage at 48 hours (Fig. 4B). This was reflected in a significant time-dependent difference in inhibition of cell viability also starting at 48 hours (Fig. 4C). The higher potency of DBK-1154, when compared to NZ-8-061, was also seen across patient-derived primary glioma cells in which DBK-1154 induced complete inhibition of cell viability (Fig. 4E). Importantly, using the same cell culture conditions, most of the tested kinase inhibitors did not reach even 50\% reduction in cell viability with up to $10 \mu \mathrm{M}$ concentration in all five of the tested patient-derived primary glioma cells (Supplementary Fig. 6).

Next, we proceeded to test DBK-1154 in the same intracranial E98 model as with NZ-8-061. To compensate for the lower oral bioavailability of DBK-1154, it was dosed at $100 \mathrm{mg} / \mathrm{kg}$, twice daily, using the same homogeneous formulation, with expected transient brain exposure of about 8 micromolar twice a day. This level of CNS exposure is roughly comparable to the IC50 for colony formation for DBK-1154 in vitro (Fig. 4D). DBK-1154 therapy caused tumor growth reduction after day 5 (Supplementary Fig. 8D), and reaching statistically significant difference at day 14 (Fig. 4F). Further, by using a linear mixed effects model that pooled the control groups from both experiments with the assumption that only the starting luciferase signals differ, the effects with DBK-1154 on linear tumor growth were found to be highly significant $(\mathrm{p}=0.00316$, Bonferroni $\mathrm{p}=0.00632$ ). Further, while NZ-8-061 monotherapy did not increase the survival of the intracranial tumor bearing mice (Supplementary figure 7A), the higher in vitro efficiency and in vivo brain penetrance of DBK-1154 translated to a significant, almost 2-fold longer overall mouse survival. The median survival was 15 days for the control group, and 26 days for the DBK-1154 
treated group ( $\mathrm{p}=0.012$ ) (Fig. 4G). Together these data indicate that of the studied SMAPs, DBK1154 has a greater potential as a GB therapy candidate molecule.

Considering the potential future development of DBK-1154 derivatives for GB therapy, DBK1154 was evaluated in an acute rat pilot (non-GLP) toxicology study at doses up to $800 \mathrm{mg} / \mathrm{kg}$ daily. Consistently with previous SMAP in vivo studies (Sangodkar et al., 2017; Kauko et al., 2018; McClinch et al., 2018), no significant body weight loss, deaths, or adverse behavioral or neurological effects were observed. The major morphologic finding was hepatocellular hypertrophy (panlobular) in all test article-treated groups, with increased severity as the dose level increased. This observation was likely related to compound specific pregnane $\mathrm{X}$ receptor (PXR) agonist activity, and was considered an adaptive rather than toxic effect. These findings indicate for a clear therapeutic window between normal and cancer cells in vivo. 
A

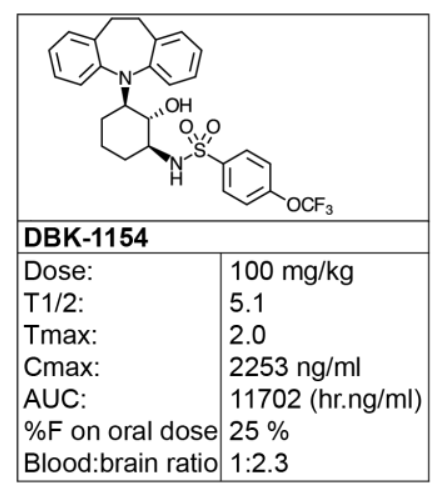

C

- DBK-1154 (13 $\mu \mathrm{M})$

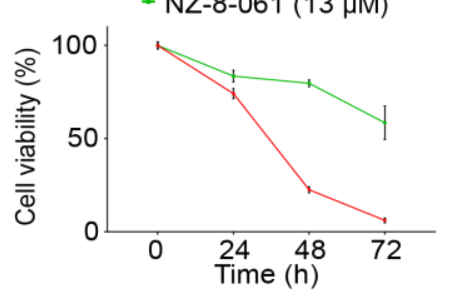

$\mathrm{F}$

E98 intracranial xenograft, day 14

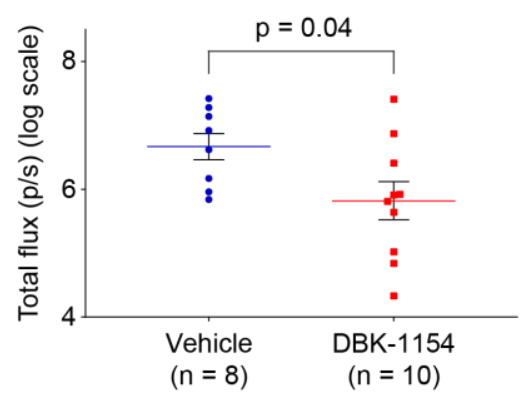

$\mathrm{E}$

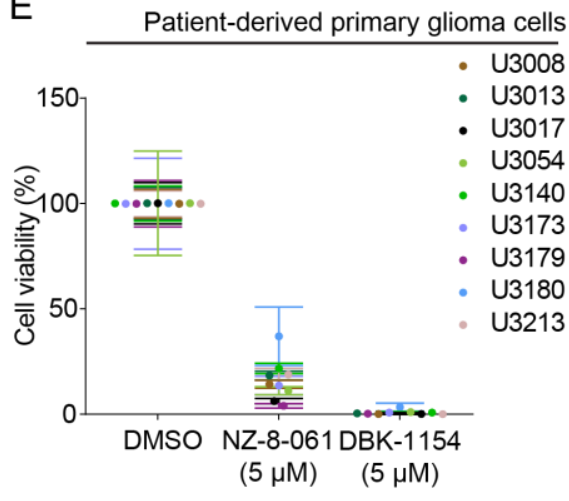

B

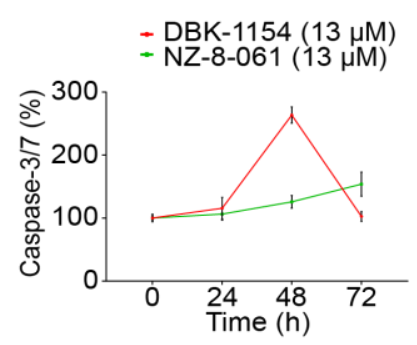

D

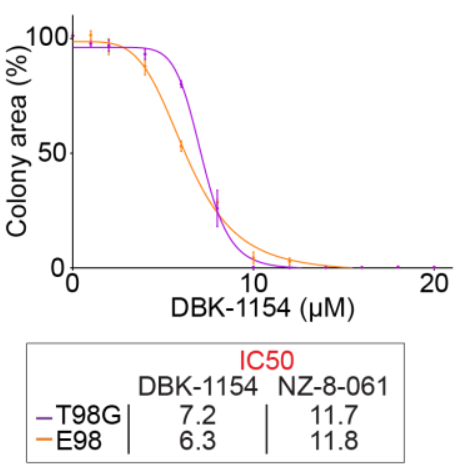

G

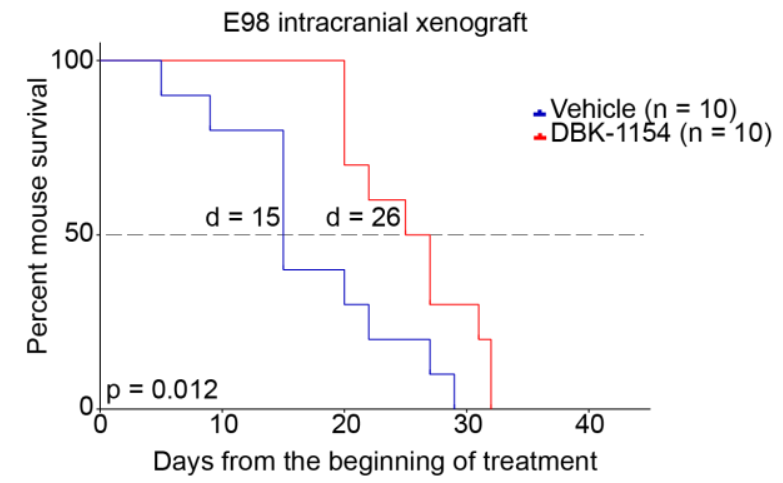

Figure 4

Figure 4: Oral dosing of DBK-1154 increases survival of mouse with intracranial infiltrative GB xenograft

A) Mouse in vivo pharmacokinetic parameters (T⿺𠃊1/2 hr, Tmax hr, Cmax ng/mL, AUC hr.ng/mL, CL mL/hr/kg, \%F and blood-brain ratio) after $100 \mathrm{mg} / \mathrm{kg}$ dosage via p.o. of DBK-1154. B) Time-dependent Caspase -3/7 activation in E98 cells, after either DT-061 or DBK-1154 treatment (13 $\mu \mathrm{M})$. C) Parallel with the caspase 3/7 assay, a cell viability assay was run with same timepoints and concentration. Data shown are means from 6 replicates \pm SD. D) Colony growth reduction after DBK-1154 treatment in GB cell lines in indicated concentrations. IC50 comparison between DBK-1154 and NZ-8-061 is shown in the insert. Data shown are means from 4 replicates \pm SD. E) Cell viability inhibition after DBK-1154 $(5 \mu \mathrm{M})$ or NZ-8-061 $(5 \mu \mathrm{M})$ treatment in patient-derived primary glioma cells cultured in serum-free neural stem cell medium. Data shown are means from 6 replicates \pm SD. F) Bioluminescence comparison on the day 14 during the orthotopic E98 in vivo model between vehicle or $100 \mathrm{mg} / \mathrm{kg}$ DBK-1154 treatment. Data shown are means from 8 vehicle and 10 DBK-1154 treated mice \pm SEM, *, p-value by Student's t-test. G) Survival of mice with orthotopic E98 xenografts after vehicle or $100 \mathrm{mg} / \mathrm{kg}$ DBK-1154 treatment, ${ }^{*}, \mathrm{P}<0.05$ by Gehan-Breslow-Wilcoxon test. Mice were randomized to 2 groups of 10 mice each based on bioluminescence signal before starting the treatment. Median survival was increased with DBK-1154 treatment from 15 days to 26 days. 
Collectively these results provide proof-of-principle evidence for preclinical in vivo efficacy, and acceptable safety profile of SMAPs as novel candidate class of BBB penetrable, tumor suppressor reactivation therapeutics. Notably, the efficacy of SMAPs did not depend on the subtype of GB, or the genomic alterations, in either established GB cell lines or in patient derived primary glioma cells. Moreover, SMAPs were found to be superior to a range of kinase inhibitors in their capacity to kill patient derived primary glioma cells. These results provide the first indications that PP2A reactivation might be able to challenge the current paradigm in GB therapies which has been strongly focused on targeting specific genetically altered cancer drivers with highly specific inhibitors (Verhaak et al., 2010; Barretina et al., 2012; Brennan et al., 2013). PP2A is known to simultaneously target a number of cancer driver pathways and pro-apoptotic mechanisms (Perrotti and Neviani, 2013; Kauko and Westermarck, 2018). We envision that these wide-spectrum effects may explain the very robust preclinical survival effects across kinase inhibitor resistant cell lines harboring heterogenous genetic drivers.

Another serious obstacle for development of GB therapies is inadequate exposure of most developed molecules in the CNS due to the BBB (Harder et al., 2018). Here we show that both SMAPs have retained the BBB penetration properties of tricyclics from which they were derived. This was not obvious as SMAPs contain a significant modification of the original tricyclics with loss of a polar amine salt and addition of sulfonamide and hydroxyl moieties. The observation that out of the studied SMAPs, DBK-1154 preferably partitions into the brain, can be explained by its lower tPSA and higher cLopP. Consequently, this better brain partitioning can partly explain the significant almost 2-fold prolongation of survival of mice with DBK-1154 versus NZ-8-061. In addition to GB, poor small molecule BBB penetration is a serious problem also for the therapy of 
brain metastasis from many non-CNS cancer types. As these are more common than primary brain tumors such as GB, the presented proof-of-principle data for usefulness of SMAPs as CNS therapeutics might be relevant for much larger patient population than only GB patients.

In addition to their different in vivo brain penetrance properties, NZ-8-061 seems to have cytostatic effects at the doses tested, whereas DBK-1154 most probably induces both, decreased proliferation and cell death. On the other hand, a SMAP derivative DBK-766 defective in PP2A reactivation (Sangodkar et al., 2017; McClinch et al., 2018), failed to suppress GB cell viability. The intracellular pathways involved in the apoptosis induction by DBK-1154 in vitro were not addressed in this concise communication, but clearly remains as an important future question to be addressed. As such, the results provide a clear indication that chemical structure of DBK-1154 could serve as a scaffold for further development of even more potent small molecule activators of PP2A for GB therapy. Following this rationale, a medicinal chemistry program identifying DBK-1154 analogs with improved oral bioavailability is currently ongoing.

PP2A activity is known to modulate kinase inhibitor responses in hematological and solid cancers (Neviani et al., 2013; Kaur et al., 2016; Kauko et al., 2018). In vivo, combination studies with SMAPs demonstrated significant tumor regression in KRAS-mutant lung cancer xenograft model with MEK inhibitors (Kauko et al., 2018). Therefore, the results reported here not only serve as a proof-of-principle for the feasibility of SMAPs as novel glioblastoma therapeutics, but also pave the way for future combination studies with kinase inhibitors, and potentially also other type of therapies in brain cancers. As PP2A inhibition is also one of the pathogenic mechanisms in Alzheimer's disease, our data demonstrating BBB penetration and in vivo therapeutic effects of 
SMAPs in intracranial model might also serve as a landmark for use of SMAPs also in other CNS pathologies.

\section{Methods}

Further information is provided in Supplemental Methods.

Data availability statement: Data is available from the corresponding author Jukka Westermarck (jukka.westermarck@utu.fi) upon request.

Funding information: Project was funded by Jane and Aatos Erkko foundation, Sigrid Juselius Foundation, and Finnish Cancer Foundation.

Conflict of interests: The Icahn School of Medicine at Mount Sinai has filed patents covering composition of matter on the small molecules disclosed herein for the treatment of human cancer and other diseases (International Application Numbers: PCT/US15/19770, PCT/US15/19764; and US Patent: US 9,540,358 B2). Mount Sinai is actively seeking commercial partners for the further development of the technology. D.B.K., M.O., G.N. has a financial interest in the commercialization of the technology. Other authors declare no conflicts of interests.

\section{References}

Barretina J, Caponigro G, Stransky N, Venkatesan K, Margolin AA, Kim S, et al. The Cancer Cell Line Encyclopedia enables predictive modelling of anticancer drug sensitivity. Nature 2012; 483(7391): 603-7. 
Brennan CW, Verhaak RG, McKenna A, Campos B, Noushmehr H, Salama SR, et al. The somatic genomic landscape of glioblastoma. Cell 2013; 155(2): 462-77.

Claes A, Schuuring J, Boots-Sprenger S, Hendriks-Cornelissen S, Dekkers M, van der Kogel AJ, et al. Phenotypic and genotypic characterization of orthotopic human glioma models and its relevance for the study of anti-glioma therapy. Brain Pathol 2008; 18(3): 423-33.

Dunn GP, Rinne ML, Wykosky J, Genovese G, Quayle SN, Dunn IF, et al. Emerging insights into the molecular and cellular basis of glioblastoma. Genes Dev 2012; 26(8): 756-84.

Harder BG, Blomquist MR, Wang J, Kim AJ, Woodworth GF, Winkles JA, et al. Developments in Blood-Brain Barrier Penetrance and Drug Repurposing for Improved Treatment of Glioblastoma. Frontiers in oncology 2018; 8: 462.

Kastrinsky DB, Sangodkar J, Zaware N, Izadmehr S, Dhawan NS, Narla G, et al. Reengineered tricyclic anti-cancer agents. Bioorg Med Chem 2015; 23(19): 6528-34.

Kauko O, O'Connor CM, Kulesskiy E, Sangodkar J, Aakula A, Izadmehr S, et al. PP2A inhibition is a druggable MEK inhibitor resistance mechanism in KRAS-mutant lung cancer cells. Science translational medicine 2018; 10(450).

Kauko O, Westermarck J. Non-genomic mechanisms of protein phosphatase 2A (PP2A) regulation in cancer. Int J Biochem Cell Biol 2018; 96: 157-64.

Kaur A, Denisova OV, Qiao X, Jumppanen M, Peuhu E, Ahmed SU, et al. PP2A Inhibitor PME1 Drives Kinase Inhibitor Resistance in Glioma Cells. Cancer Research 2016; 76(23): 7001-11. Kelder J, Grootenhuis PD, Bayada DM, Delbressine LP, Ploemen JP. Polar molecular surface as a dominating determinant for oral absorption and brain penetration of drugs. Pharm Res 1999; 16(10): 1514-9. 
Laajala TD, Jumppanen M, Huhtaniemi R, Fey V, Kaur A, Knuuttila M, et al. Optimized design and analysis of preclinical intervention studies in vivo. Sci Rep 2016; 6: 30723.

Le Joncour V, Karaman S, Laakkonen PM. Predicting In Vivo Payloads Delivery using a Bloodbrain Tumor-barrier in a Dish. J Vis Exp 2019(146).

McClinch K, Avelar RA, Callejas D, Izadmehr S, Wiredja D, Perl A, et al. Small-Molecule Activators of Protein Phosphatase 2A for the Treatment of Castration-Resistant Prostate Cancer. Cancer Research 2018; 78(8): 2065-80.

Mooney J, Bernstock JD, Ilyas A, Ibrahim A, Yamashita D, Markert JM, et al. Current approaches and challenges in the molecular therapeutic targeting of glioblastoma. World Neurosurg 2019.

Narla G, Sangodkar J, Ryder CB. The impact of phosphatases on proliferative and survival signaling in cancer. Cell Mol Life Sci 2018; 75(15): 2695-718.

Navis AC, van Lith SA, van Duijnhoven SM, de Pooter M, Yetkin-Arik B, Wesseling P, et al. Identification of a novel MET mutation in high-grade glioma resulting in an auto-active intracellular protein. Acta Neuropathol 2015; 130(1): 131-44.

Neviani P, Harb JG, Oaks JJ, Santhanam R, Walker CJ, Ellis JJ, et al. PP2A-activating drugs selectively eradicate TKI-resistant chronic myeloid leukemic stem cells. J Clin Invest 2013; 123(10): 4144-57.

Perrotti D, Neviani P. Protein phosphatase 2A: a target for anticancer therapy. The lancet oncology 2013; 14(6): e229-38.

Sangodkar J, Perl A, Tohme R, Kiselar J, Kastrinsky DB, Zaware N, et al. Activation of tumor suppressor protein PP2A inhibits KRAS-driven tumor growth. J Clin Invest 2017; 127(6): 208190. 
Tomiyama A, Ichimura K. Signal transduction pathways and resistance to targeted therapies in glioma. Semin Cancer Biol 2019.

Tomiyama A, Kobayashi T, Mori K, Ichimura K. Protein Phosphatases-A Touchy Enemy in the Battle Against Glioblastomas: A Review. Cancers (Basel) 2019; 11(2).

van den Heuvel C, Navis AC, de Bitter T, Amiri H, Verrijp K, Heerschap A, et al. Selective MET Kinase Inhibition in MET-Dependent Glioma Models Alters Gene Expression and Induces Tumor Plasticity. Mol Cancer Res 2017; 15(11): 1587-97.

Verhaak RG, Hoadley KA, Purdom E, Wang V, Qi Y, Wilkerson MD, et al. Integrated genomic analysis identifies clinically relevant subtypes of glioblastoma characterized by abnormalities in PDGFRA, IDH1, EGFR, and NF1. Cancer Cell 2010; 17(1): 98-110.

Wang Q, Hu B, Hu X, Kim H, Squatrito M, Scarpace L, et al. Tumor Evolution of Glioma-Intrinsic Gene Expression Subtypes Associates with Immunological Changes in the Microenvironment. Cancer Cell 2017; 32(1): 42-56 e6.

Xie Y, Bergstrom T, Jiang Y, Johansson P, Marinescu VD, Lindberg N, et al. The Human Glioblastoma Cell Culture Resource: Validated Cell Models Representing All Molecular Subtypes. EBioMedicine 2015; 2(10): 1351-63. 